\title{
An Improved Approximation Algorithm for the Asymmetric TSP with Strengthened Triangle Inequality
}

\author{
Markus Bläser* $\quad$ Bodo Manthey ${ }^{\dagger} \quad$ Jiří Sgall ${ }^{\ddagger}$
}

\begin{abstract}
We consider the asymmetric traveling salesperson problem with $\gamma$-parameterized triangle inequality for $\gamma \in[1 / 2,1)$. That means, the edge weights fulfill $w(u, v) \leq$ $\gamma \cdot(w(u, x)+w(x, v))$ for all nodes $u, v, x$. Chandran and Ram (Proc. 19th STACS, LNCS 2285, pp. 227-237, 2002) gave the first constant factor approximation algorithm with polynomial running time for this problem. They achieve performance ratio $\gamma /(1-\gamma)$. We devise an approximation algorithm with performance ratio $(1+\gamma) /\left(2-\gamma-\gamma^{3}\right)$, which is better for $\gamma \in[0.5437,1)$, that is, for the particularly interesting large values of $\gamma$.
\end{abstract}

\section{Introduction}

The traveling salesperson problem is a well-known NP optimization problem. Given a complete loopless graph $G$ and a weight function $w$ that assigns to each edge a nonnegative weight, our goal is to find a tour of minimum weight that visits each node exactly once (i.e., a Hamiltonian tour of minimum weight). In general, the graph $G$ may be directed. In this case, one also speaks of the asymmetric traveling salesperson problem (ATSP). An important and well-studied special case is the case where $w$ is symmetric (TSP), that is, $w(u, v)=w(v, u)$ for all $u, v \in V$. In other words, the underlying graph can be considered undirected.

TSP and henceforth ATSP are both NPO-complete. Thus there is no good approximation algorithm for these two problems, unless $N P=P$. A natural restriction is that the weight function $w$ should fulfill the triangle inequality

$$
w(u, v) \leq w(u, x)+w(x, v) \quad \text { for all } u, x, v \in V .
$$

We call the corresponding problems $\Delta$-ATSP and $\Delta$-TSP in the asymmetric and symmetric case, respectively. For $\Delta$-TSP, Christofides [10] devised a $3 / 2$ approximation algorithm with polynomial running time, whereas the best approximation algorithm for $\Delta$-ATSP has only performance ratio $\log n$ as was shown by Frieze, Galbiati, and Maffioli [11]. This was improved by Bläser [5] and Kaplan et al. [12] to $0.999 \cdot \log n$ and $0.841 \cdot \log n$, respectively. Many researchers conjecture that there is a constant factor approximation algorithm also for $\Delta$ ATSP, but this question is still open after more than two decades.

\footnotetext{
${ }^{*}$ Institut für Theoretische Informatik, ETH Zürich, 8092 Zürich, Switzerland, mblaeser@inf .ethz.ch. Partially supported by DFG research grant Bl 511/5-1.

${ }^{\dagger}$ Institut für Theoretische Informatik, Universität zu Lübeck, Ratzeburger Allee 160, 23538 Lübeck, Germany, manthey@tcs.uni-luebeck.de. Supported by DFG research grants Re 672/3 and Bl 511/5-1.

${ }^{\ddagger}$ Mathematical Institute, AS CR, Žitná 25, CZ-11567 Praha 1, Czech Republic. sgalı@math.cas.cz. Partially supported by Institutional Research Plan No. AV0Z10190503, by Institute for Theoretical Computer Science, Prague (project 1M0021620808 of MŠMT ČR) and by grant IAA1019401 of GA AV ČR.
} 
Here we consider a strengthening of the triangle inequality (1), which allows a constant factor approximation: Let $\gamma$ be some constant with $1 / 2 \leq \gamma<1$. An instance of the problem $\Delta(\gamma)$-ATSP is a complete loopless directed graph $G$ with node set $V$ and a weight function $w$ assigning to each edge of $G$ a nonnegative weight. The weight function fulfills the $\gamma$ parameterized triangle inequality, i.e.,

$$
w(u, v) \leq \gamma \cdot(w(u, x)+w(x, v)) \quad \text { for all } u, x, v \in V .
$$

The goal is to compute a TSP tour of minimum weight.

One can also view the $\gamma$-parameterized triangle inequality as a data dependent bound. Given an instance of $\Delta$-(A)TSP, we can compute $\tilde{\gamma}=\max \{w(u, v) /(w(u, x)+w(x, v))\}$ and use an algorithm for $\Delta(\tilde{\gamma})-(\mathrm{A}) \mathrm{TSP}$ to obtain better performance guarantees on instances where $\tilde{\gamma}$ is small enough.

\subsection{Previous and new results}

As mentioned above, for $\Delta$-ATSP and $\Delta$-TSP, there are approximation algorithms with polynomial running time achieving performance ratios $\log n$ and $3 / 2$, respectively.

Böckenhauer et al. [7] studied the symmetric traveling salesperson problem with $\gamma$ parameterized triangle inequality for $\gamma \in[1 / 2,1)$. They achieve approximation performance $\min \left\{1+(2 \gamma-1) /\left(3 \gamma^{2}-2 \gamma+1\right), 2 / 3+\gamma /(3-3 \gamma)\right\}$. Then Böckenhauer et al. [8] (improving a result by Andreae and Bandelt [3]) as well as Bender and Chekuri [4] and Andreae [2] considered the symmetric case with $\gamma$-parameterized triangle inequality for $\gamma \geq 1$. Combining their results, we get an approximation algorithm with performance guarantee $\min \left\{3 \gamma^{2} / 2, \gamma^{2}+\gamma, 4 \gamma\right\}$.

Chandran and Ram [9] studied the asymmetric traveling salesperson problem with $\gamma$ parameterized triangle inequality for $\gamma \in[1 / 2,1)$. They designed a constant factor approximation algorithm with performance ratio (asymptotically) $\gamma /(1-\gamma)$, in contrast to the $\log n$ upper bound for $\Delta$-ATSP. Since in the asymmetric case we even do not know whether for $\gamma=1$ an approximation algorithm with constant performance ratio exists, studying the case $\gamma \geq 1$ does not look very promising at the moment.

As our main result, we present an approximation algorithm SHORTCUT with performance ratio $(1+\gamma) /\left(2-\gamma-\gamma^{3}\right)$. This improves the result by Chandran and Ram for $\gamma \in[0.5437,1)$, that is, for the particularly interesting large values of $\gamma$. The running time of our algorithm is $O\left(n^{3}\right)$, which matches the running time of the algorithm by Chandran and Ram. Our new algorithm also improves upon the conference version of this paper [6] where an approximation ratio of $2 /\left(2-\gamma-\gamma^{3}\right)$ is obtained.

The approximation performance of SHORTCUT is better than the one obtained by Chandran and Ram [9], if

$$
\frac{\gamma}{1-\gamma} \geq \frac{1+\gamma}{2-\gamma-\gamma^{3}}=\frac{1+\gamma}{(1-\gamma)\left(2+\gamma+\gamma^{2}\right)}
$$

For $\gamma<1$ this is equivalent to $\gamma+\gamma^{2}+\gamma^{3} \geq 1$. The only real valued root of the corresponding polynomial can be computed exactly, it is $X / 3-2 /(3 X)-1 / 3$, where $X=(17+3 \sqrt{33})^{1 / 3}$; the numerical value of the root is approximately 0.5437 . In particular, SHORTCUT is better for $\gamma \in[0.5437,1)$. For $\gamma$ close to 1 , the improvement is by a factor close to 2 . Figure 1 compares the performances in dependence on $\gamma$. 

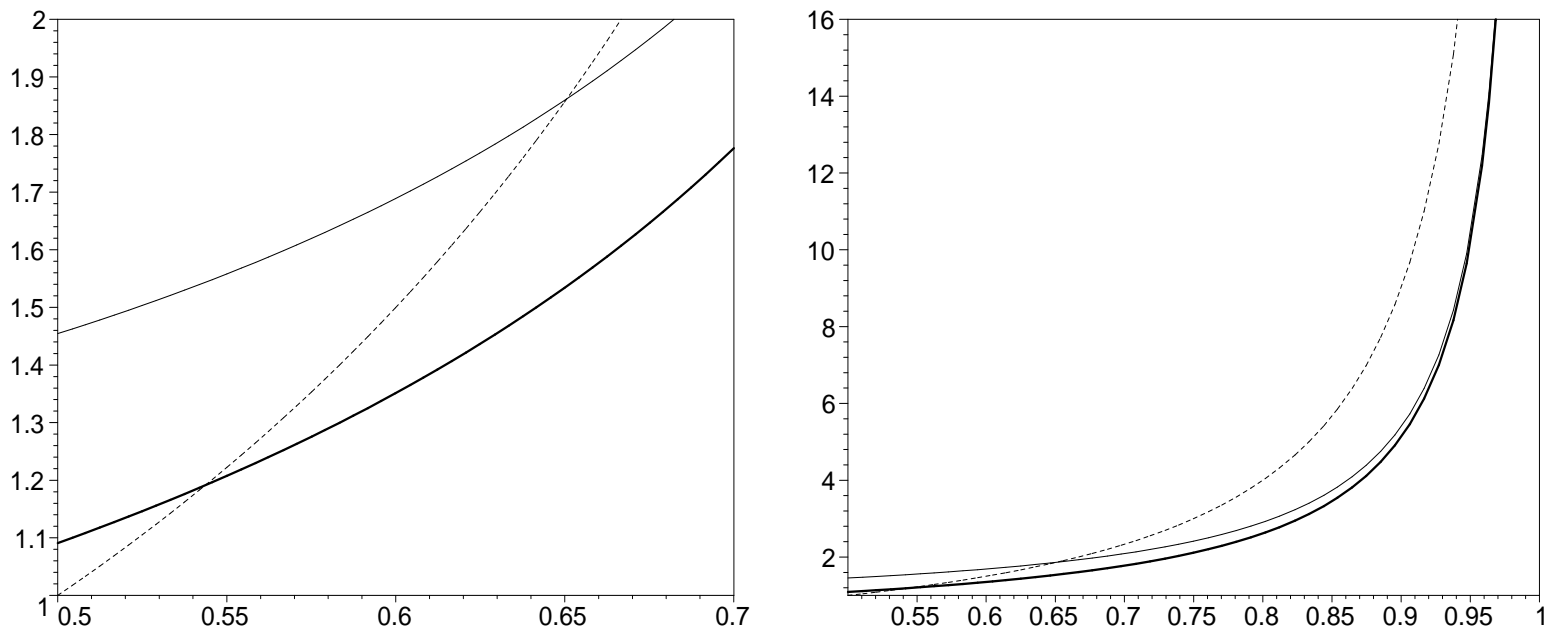

Figure 1: The approximation performance of the algorithm SHORTCUT (drawn thick) compared to the one by Chandran and Ram [9] (drawn dashed) and Bläser [6] (drawn thin).

\subsection{Notations and conventions}

For a set of nodes $V$, let $K(V)$ denote the set of edges $(V \times V) \backslash\{(v, v) \mid v \in V\}$. Throughout this work, we are considering directed graphs $G=(V, K(V))$ together with a weight function $w: K(V) \rightarrow \mathbb{Q}_{\geq 0}$ and a parameter $\gamma \in[1 / 2,1)$. We always require that $w$ fulfills the $\gamma$ parameterized triangle inequality (2). (Note that if $w$ fulfills the $\gamma$-parameterized triangle inequality for some $\gamma$, then necessarily $\gamma \geq 1 / 2$. Thus the lower bound is no restriction.)

A cycle cover of a directed graph $G$ is a spanning subgraph that consists solely of node disjoint directed cycles. For any subgraph $S=(V, E)$ of $G$, the weight $w(S)$ of $S$ is defined as the sum of the weights of the edges in $E$, that is, $w(S)=\sum_{e \in E} w(e)$. In particular, this defines the weight of cycle covers and TSP tours.

For a given directed graph $G$ with weight function $w$, let $\mathrm{AB}(G)$ denote the weight of a minimum weight cycle cover. (This is also called the assignment bound.) Furthermore, let $\operatorname{TSP}(G)$ denote the weight of a minimum weight TSP tour of $G$. Obviously, we have $\mathrm{AB}(G) \leq \operatorname{TSP}(G) \cdot \operatorname{AB}(G)$ and a corresponding minimum weight cycle cover can be computed in time $O\left(n^{3}\right)$; there are various algorithms with this time bound, for instance based on bipartite matching (see [1] for an overview).

\section{The approximation algorithm}

Our new approximation algorithm SHORTCUT is based on the repeated cycle cover approach by Frieze, Galbiati, and Maffioli [11]. The algorithm of Frieze, Galbiati, and Maffioli without any modifications already yields a $1 /(1-\gamma)$ approximation for $\Delta(\gamma)$-ATSP, as noticed by Bläser [6].

We sketch the improved algorithm now; an illustration is given in Figure 2 (using notation for vertices later introduced in the algorithm). First a minimum weight cycle cover $C$ is computed. The nodes are split into two sets $V_{1}$ and $V_{2}$ so that the two sets alternate along in each cycle of $C$ (to be precise, in case of an odd cycle, two nodes of $V_{1}$ are adjacent). Then 
we recursively compute two TSP tours $T_{1}$ and $T_{2}$, one in the graph $G_{1}$ induced by $V_{1}$ (i.e., $G_{1}=\left(V_{1}, K\left(V_{1}\right)\right)$ with weight function $w_{1}$ where $w_{1}$ is the restriction of $w$ to $\left.K\left(V_{1}\right)\right)$ and the other one in the graph $G_{2}$ induced by $V_{2}$. Finally, we combine $C$ and one of $T_{p}$ into an Eulerian tour and obtain the resulting Hamiltonian tour $T$ by taking shortcuts.

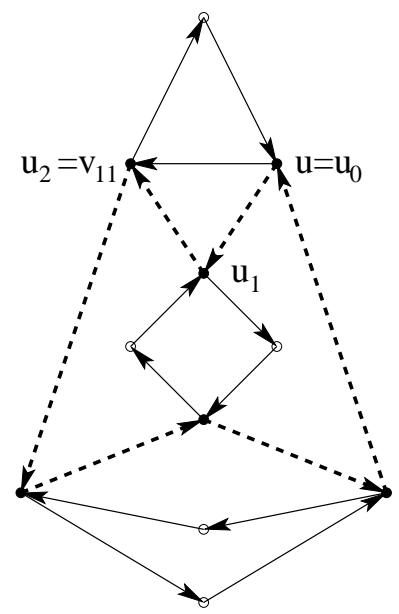

(a)

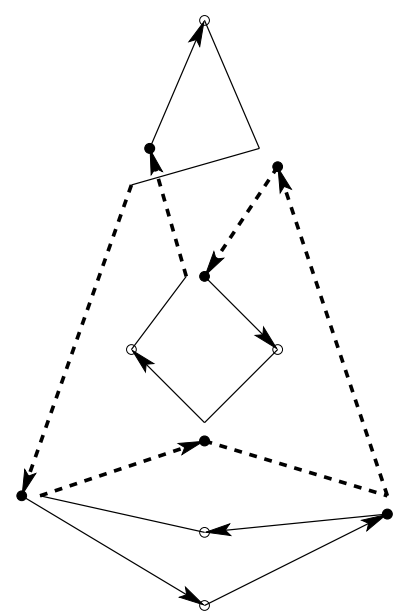

(c)

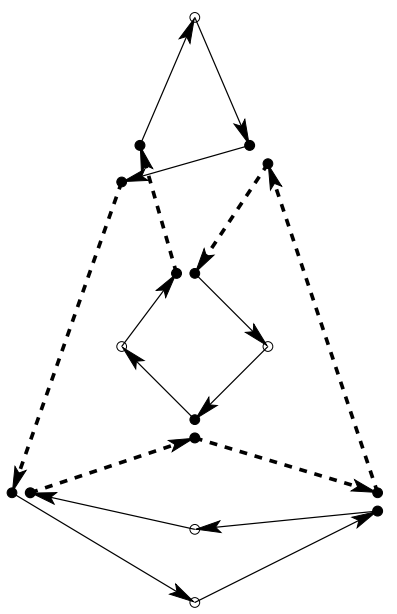

(b)

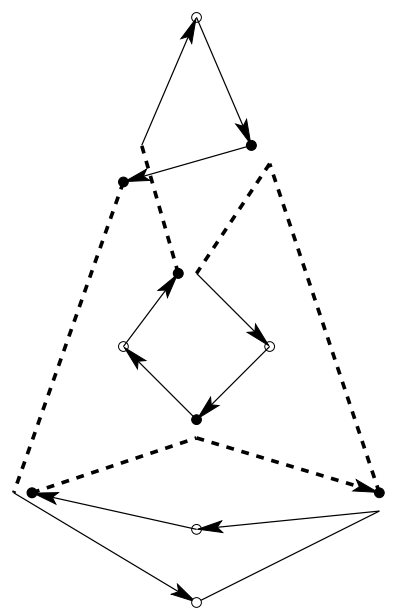

(d)

Figure 2: An example of a run of SHORTCUT. (a) A graph with nodes of $V_{1}$ drawn as solid bullets, edges of $C$ drawn as solid arrows, and edges of $T_{1}$ drawn dashed. (Edges of $T_{2}$ and unused edges are omitted.) (b) The Eulerian tour $E_{1}$ drawn with nodes from $V_{1}$ duplicated. (c) and (d): The Hamiltonian tours $H_{11}$ and $H_{12}$, respectively; the bullets and arrows at the nodes skipped by shortcuts are omitted.

Taking shortcuts is the place where we use the power of the strengthened triangle inequality. Suppose that walk $S^{\prime}$ is obtained by taking shortcuts from another walk $S$. Even with regular triangle inequality, we have $w\left(S^{\prime}\right) \leq w(S)$. The additional power of the $\gamma$-parameterized triangle inequality is used when we bound $w\left(S^{\prime}\right)$ by the sum of the contributions of (the occurrences of) the edges $e \in S$. If the edge $e$ appears in $S^{\prime}$, its contribution is $w(e)$. If the edge 
$e$ is contracted at least once, i.e., one of its endpoints is omitted, its contribution is bounded by $\gamma \cdot w(e)$. If the edge $e$ is contracted at least twice - for example, if both of its endpoints are omitted - its contribution further decreases and is bounded by $\gamma^{2} w(e)$.

For each $T_{p}$, the corresponding Eulerian tour contains two occurrences of each vertex in $V_{p}$ and a single occurrence of the other vertices. For each vertex we may omit either one of the two occurrences. We actually generate two candidate Hamiltonian tours (for each $T_{p}$ ), so that at each vertex $V_{p}$, each of the two ways of arranging shortcuts is used once. At the end we output the shortest tour of the four candidates.

To get the best approximation factors we need to arrange the shortcuts quite carefully. If each cycle has two vertices, this can be described easily: The Eulerian tour always takes one edge of $T_{p}$, then goes around a cycle of $T$, and so on. In one candidate Hamiltonian tour we skip each vertex in $V_{p}$ upon entering the corresponding cycle, while in the other candidate, we skip it upon leaving the cycle. The result is that each edge of $T_{p}$ is contracted in both candidate tours, while each edge of $C$ is contracted in exactly one of the two candidate tours. In the general case, we arrange the shortcuts along $T_{p}$ so that each edge of $T_{p}$ is contracted in both candidates; this is possible with the exception of one edge. This one edge can be contracted twice in one candidate tour and not contracted at all in the other candidate. It turns out that if this edge is short, this does not hurt us, see Lemma 2.2. A similar problem can occur with the edge of an odd cycle which connects two vertices of $V_{1}$; again, choosing it to be a short edge, Lemma 2.2 solves this problem. Note that the example in Figure 2 contains both of these types of problematic edges, namely $\left(u_{0}, u_{1}\right)$ in $T_{p}$ and $\left(u_{0}, u_{2}\right)$ in an odd cycle of $C$.

\section{Algorithm SHORTCUT \\ Input: directed graph $G=(V, K(V))$ with weight function $w$ where $w$ fulfills the $\gamma$-parameterized triangle inequality (2) for some $\gamma \in[1 / 2,1)$}

\section{Output: TSP tour $T$}

1. Compute a minimum weight cycle cover $C$ of $G$. If $C$ has a single cycle, let $T=C$ and stop.

2. Let $C_{1}, \ldots, C_{t}$ be the cycles of $C$. Denote the nodes in $C_{i}$ by $v_{i 1}, v_{i 2}, \ldots, v_{i k_{i}}=$ $v_{i 0}$, in the order along the cycle starting so that the edge $\left(v_{i 0}, v_{i 1}\right)$ has the minimal weight of all the edges of $C_{i}$.

Let $V_{1}=\left\{v_{i j} \mid i=1, \ldots, t, j\right.$ odd $\}$ and $V_{2}=\left\{v_{i j} \mid i=1, \ldots, t, j>0\right.$ even $\}$. Furthermore, let $\bar{V}_{1}=\left\{v_{i 1} \mid i=1, \ldots, t\right\}$ and $\bar{V}_{2}=\left\{v_{i 2} \mid i=1, \ldots, t\right\}$. Note that $V$ is a disjoint union of $V_{1}$ and $V_{2}$; furthermore, each $\bar{V}_{p}$ has exactly one node in each cycle of $C$.

3. Recursively compute two TSP tours $T_{1}$ and $T_{2}$ of the graphs $G_{1}$ and $G_{2}$ that are induced by $V_{1}$ and $V_{2}$.

4. For each $p \in\{1,2\}$, construct an Eulerian tour $E_{p}$ of $\left(V, C \cup T_{p}\right)$ as follows. Visit the nodes of $V_{p}$ in the order given by $T_{p}$. For each node $v \in \bar{V}_{p}$, the tour runs through the (unique) cycle in $C$ that $v$ belongs to; after that, it continues with the next node of $T_{p}$. For $v \in V_{p} \backslash \bar{V}_{p}$, the tour continues immediately with the next node of $T_{p}$.

Note that each node of $V_{p}$ occurs exactly twice on $E_{p}$ and each node of $V-V_{p}$ occurs exactly once. 
5. For each $p \in\{1,2\}$, construct two Hamiltonian tours $H_{p 1}$ and $H_{p 2}$ from $E_{p}$ by taking shortcuts. To do this, we need to determine for each $u \in V_{p}$ which of its two occurrences in $E_{p}$ is removed. There are two possibilities for each node, we make sure that each of $H_{p q}$ uses a different one. In addition, we choose a special way to do it:

(a) First, we choose a starting node $u_{1}$ as follows: If $V_{p}=\bar{V}_{p}$, let $u_{1}$ be an arbitrary node from $V_{p}$. Otherwise let $u$ be an arbitrary node from $V_{p} \backslash \bar{V}_{p}$; choose $\left(u_{0}, u_{1}\right)$ to be the lighter edge of the two edges of $T_{p}$ incident with $u$ (i.e., $u=u_{0}$ or $u=u_{1}$ ). Denote the nodes of $V_{p}$ by $u_{1}, u_{2}, \ldots, u_{k}=u_{0}$ in the order along the tour $T_{p}$.

(b) For $u_{1}$, do the following: In $H_{p 1}$, omit (i.e., take a shortcut at) the occurrence of $u_{1}$ where $E_{p}$ continues by an edge from $T_{p}$ (i.e., the next node in $E_{p}$ is $\left.u_{2}\right)$; in $H_{p 2}$ omit the occurrence of $u_{1}$ where $E_{p}$ continues along $C$.

(c) Process the nodes $u_{i}$, for $i=2, \ldots, k$ as follows. Consider the consecutive occurrences of $u_{i-1}$ and $u_{i}$ in $E_{p}$ (i.e., the place when $E_{p}$ traverses the edge $\left.\left(u_{i-1}, u_{i}\right)\right)$. Let $H_{p q}$ be the tour where this occurrence of $u_{i-1}$ was not skipped. Skip the occurrence of $u_{i}$ following $u_{i-1}$ in $H_{p q}$ and skip the other occurrence of $u_{i}$ in $H_{p, 3-q}$.

6. Output as $T$ the shortest tour of the four tours $H_{p q}$.

The next lemma uses the analysis of shortcuts to bound the weight of a minimum weight TSP tour of $G_{1}$ and $G_{2}$ in terms of the weight of a minimum weight TSP tour of $G$.

Lemma 2.1 Let $V_{1}, V_{2} \subseteq V$ be two disjoint sets of nodes. Let $G_{1}$ and $G_{2}$ be the graphs induced by $V_{1}$ and $V_{2}$, respectively. Then

$$
\operatorname{TSP}\left(G_{1}\right)+\operatorname{TSP}\left(G_{2}\right) \leq\left(1+\gamma^{2}\right) \operatorname{TSP}(G) .
$$

Proof. Let $T$ be a minimum weight TSP tour of $G$. Thus $w(T)=\operatorname{TSP}(G)$. We construct two TSP tours $T_{1}$ and $T_{2}$ of $G_{1}$ and $G_{2}$, respectively, such that $w\left(T_{1}\right)+w\left(T_{2}\right) \leq\left(1+\gamma^{2}\right) w(T)$. This proves the claim of the lemma.

Given $T$, we construct $T_{1}$ and $T_{2}$ by taking shortcuts. We move along the tour $T$ starting with an arbitrary node in $V_{1}$ or $V_{2}$, respectively. Whenever we would visit a node not in $V_{1}$ or $V_{2}$, respectively, we directly go to the next node of $T$ that is in $V_{1}$ or $V_{2}$.

Let $e=(u, v)$ be an edge of $T$. If both $u$ and $v$ belong to $V_{1}$, then the edge $e$ appears in $T_{1}$ but is contracted (at least) twice when constructing $T_{2}$. Thus $e$ contributes weight $w(e)$ to $T_{1}$ and $\gamma^{2} w(e)$ to $T_{2}$ yielding a total contribution of $\left(1+\gamma^{2}\right) w(e)$. If both $u$ and $v$ belong to $V_{2}$, the same analysis works. If $u$ belongs to $V_{1}$ and $v$ belongs to $V_{2}$ or vice versa, then $e$ is contracted at least once to obtain $T_{1}$ and at least once to obtain $T_{2}$. Thus the total contribution is at most $2 \gamma \cdot w(e) \leq\left(1+\gamma^{2}\right) w(e)$. Summing over all edges $e$ of $T$ yields the result.

Next we use a similar analysis to show that the algorithm guarantees that the total weight of the four tours $H_{p q}$ is bounded by $(2+2 \gamma) w(C)+2 \gamma\left(w\left(T_{1}\right)+w\left(T_{2}\right)\right)$. As mentioned before, in case of an odd cycle and also in case of the edge $\left(u_{0}, u_{1}\right)$ in $T_{p}$, an edge may be contracted twice in one of the four tours instead of being contracted in two distinct tours. This would multiply the weight of the edge by $1+\gamma^{2}$ instead of $2 \gamma$ and thus increase its contribution beyond the claimed bound. The next lemma gives a tighter bound in these cases. 
Lemma 2.2 Let $v, x, y, z \in V$ be such that $w(x, y) \leq \max \{w(v, x), w(y, z)\}$. Then

$$
w(v, z) \leq \gamma w(v, x)+(2 \gamma-1) w(x, y)+\gamma w(y, z) .
$$

Proof. Suppose that $w(x, y) \leq w(v, x)$; the other case is symmetric. By contracting first the node $x$ and then $y$ we have

$$
w(v, z) \leq \gamma^{2} w(v, x)+\gamma^{2} w(x, y)+\gamma w(y, z) .
$$

For any $\gamma \in[1 / 2,1)$, we have

$$
0 \leq \gamma^{2}-2 \gamma+1 \leq \gamma-\gamma^{2}
$$

as the corresponding equation has roots $1 / 2$ and 1 . Thus

$$
\left(\gamma^{2}-2 \gamma+1\right) w(x, y) \leq\left(\gamma-\gamma^{2}\right) w(v, x)
$$

and

$$
\begin{aligned}
w(v, z) & \leq \gamma^{2} w(v, x)+\gamma^{2} w(x, y)+\gamma w(y, z) \\
& \leq \gamma w(v, x)+(2 \gamma-1) w(x, y)+\gamma w(y, z) .
\end{aligned}
$$

Lemma 2.3 The algorithm SHORTCUT guarantees for any input that

$$
w\left(H_{11}\right)+w\left(H_{12}\right)+w\left(H_{21}\right)+w\left(H_{22}\right) \leq(2+2 \gamma) w(C)+2 \gamma\left(w\left(T_{1}\right)+w\left(T_{2}\right)\right) .
$$

Proof. We estimate the contribution of each edge $e \in E_{p}, p \in\{1,2\}$, to the four tours $H_{p q}$ produced by shortcuts.

First consider $e=(x, y) \in C$; such $e$ contributes to all four tours $H_{p q}$ and we shall bound its contribution by $(2+2 \gamma) w(e)$. If $x \in V_{p}$ then $e$ is contracted because of removing this occurrence of $x$ in exactly one of the tours $H_{p 1}$ and $H_{p 2}$; similarly for $y$. If these two contractions happen in different tours, the contribution of $e$ is at most $(2+2 \gamma) w(e)$ as claimed. If the two contractions happen in the same tour, it has to be the case that $x, y \in V_{p}$ for some $p$. By the choice of $V_{p}$ (step 2 of the algorithm), for some cycle $C_{i},(x, y)=\left(v_{i 0}, v_{i 1}\right)$, and thus $(x, y)$ is the lightest edge of $C_{i}$; in addition $p=1$. This means that in the tour $E_{1}$, the edge $(x, y)$ is preceded by another edge of $C_{i}$ which is larger. Therefore we can apply Lemma 2.2 and bound the contribution of $(x, y)$ to the tour with two contractions by $(2 \gamma-1) w(x, y)$ (instead of $\left.\gamma^{2}\right)$; its total contribution is then at most $(3+2 \gamma-1) w(x, y)$ as claimed. Lemma 2.2 increases the bound of the preceding edge in $C_{i}$ from $\gamma^{2}$ to $\gamma$ times its weight; however, the coefficient used in the previous analysis was $\gamma$, so the analysis for the other edges remains valid.

Next consider $e=\left(u_{i}, u_{i+1}\right) \in T_{p}$ for some $p$ and numbering of nodes in $T_{p}$ as in the step 5(a) of the algorithm; such $e$ contributes to two tours $H_{p 1}$ and $H_{p 2}$, and we shall bound its contribution by $2 \gamma \cdot w(e)$. Each of the two occurrences of a node $u_{i}$ in $E_{p}$ is contracted in exactly one tour of $H_{p 1}$ and $H_{p 2}$. If these contractions for the occurrences of $u_{i}$ and $u_{i+1}$ as the endpoints of $e$ happen in two different tours, the contribution of $e$ is at most $2 \gamma \cdot w(e)$ as claimed. If the two contractions happen in a single tour, it has to be the case 
that $e=\left(u_{0}, u_{1}\right)$, by the choices in the step $5(\mathrm{c})$ of the algorithm. Furthermore, it has to be the case that $\bar{V}_{p} \neq V_{p}$ : otherwise at every node in $V_{p}$ the tour $E_{p}$ follows the cycle $C_{i}$ before continuing on $T_{p}$ and in $H_{p 1}$ all occurrences of $u_{i}$ continuing along $E_{p}$ are contracted-thus the endpoints of $e$ cannot be both contracted in the same tour. This means that one of the endpoints of $e$ was chosen as $u \in V_{p} \backslash \bar{V}_{p}$ in step 5(a), in the tour $E_{p}$ the two edges of $T_{p}$ incident with $u$ follow each other, and $e$ is chosen as the lighter one of these two edges. Thus we can apply Lemma 2.2 (with $e$ playing the role of $(x, y)$ in the lemma) and bound the contribution of $e$ to the tour with two contractions by $(2 \gamma-1) w(e)$ (instead of $\gamma^{2}$ ); its total contribution is then at most $(1+2 \gamma-1) w(e)$ as claimed. As before, Lemma 2.2 increases the bound of one other edge in $E_{p}$ from $\gamma^{2}$ to $\gamma$ times its weight, but this does not change the previous analysis.

The lemma now follows by summing the contributions of all the edges in $C$ and both $T_{1}$ and $T_{2}$. One remaining subtle point is that an edge may appear in both $C$ and some $T_{p}$; in that case we need to sum over both of its occurrences, according to both cases above.

Now we can estimate the approximation performance of our algorithm.

Theorem 2.4 The approximation ratio of the algorithm SHORTCUT is bounded by

$$
R=\frac{1+\gamma}{2-\gamma-\gamma^{3}}
$$

The running time of the algorithm is cubic in the number of nodes.

Proof. The bound on the approximation performance is shown by induction on the number of nodes. If $C$ in the algorithm has a single cycle (which covers all graphs with at most three nodes), the output is an optimal tour.

Suppose that $C$ has more cycles. By the induction hypothesis and Lemma 2.1,

$$
w\left(T_{1}\right)+w\left(T_{2}\right) \leq R\left(\operatorname{TSP}\left(G_{1}\right)+\operatorname{TSP}\left(G_{2}\right)\right) \leq\left(1+\gamma^{2}\right) R \cdot \operatorname{TSP}(G) .
$$

The TSP tour $T$ computed in step 6 has weight at most

$$
\begin{aligned}
w(T) & \leq \frac{w\left(H_{11}\right)+w\left(H_{12}\right)+w\left(H_{21}\right)+w\left(H_{22}\right)}{4} \\
& \leq \frac{1+\gamma}{2} w(C)+\frac{\gamma}{2}\left(w\left(T_{1}\right)+w\left(T_{2}\right)\right) \\
& \leq \frac{1+\gamma}{2} \operatorname{TSP}(G)+\frac{\gamma+\gamma^{3}}{2} R \cdot \operatorname{TSP}(G) \\
& =R \cdot \operatorname{TSP}(G) .
\end{aligned}
$$

by Lemma 2.3, equation (3), and the choice of $R$. This proves the claim about the approximation performance.

Let $S(n)$ denote the worst case running time of the algorithm on instances with $n$ nodes. We have $S(1)=1$ and $S(n) \leq 2 \cdot S(2 n / 3)+O\left(n^{3}\right)$, for all $n>1$, because each instance is divided into two subproblems of size at most $2 n / 3$. The time for computing the two subinstances is dominated by the time $O\left(n^{3}\right)$ used to construct the cycle cover $C$. Solving the recurrence, we obtain $S(n)=O\left(n^{3}\right)$. 


\section{References}

[1] Ravindra K. Ahuja, Thomas L. Magnanti, and James B. Orlin. Network Flows: Theory, Algorithms, and Applications. Prentice Hall, 1993.

[2] Thomas Andreae. On the traveling salesman problem restricted to inputs satisfying a relaxed triangle inequality. Networks, 38(2):59-67, 2001.

[3] Thomas Andreae and Hans-Jürgen Bandelt. Performance guarantees for approximation algorithms depending on parameterized triangle inequalities. SIAM J. Discrete Math., 8(1):1-16, 1995.

[4] Michael A. Bender and Chandra Chekuri. Performance guarantees for the TSP with a parameterized triangle inequality. In Proc. 6th Int. Workshop on Algorithms and Data Structures (WADS), volume 1663 of Lecture Notes in Comput. Sci., pages 80-85, 1999.

[5] Markus Bläser. A new approximation algorithm for asymmetric TSP with triangle inequality. In Proc. 14th Ann. ACM-SIAM Symp. on Discrete Algorithms (SODA), pages 639-647, 2003.

[6] Markus Bläser. An improved approximation algorithm for the asymmetric TSP with strengthened triangle inequality. In Proc. 30th Int. Coll. on Automata, Languages, and Programming (ICALP), volume 2719 of Lecture Notes in Comput. Sci., pages 157-163, 2003.

[7] H.-J. Böckenhauer, J. Hromkovič, R. Klasing, S. Seibert, and W. Unger. Approximation algorithms for the TSP with sharpened triangle inequality. Inform. Process. Lett., 75(3):133-138, 2000.

[8] H.-J. Böckenhauer, J. Hromkovič, R. Klasing, S. Seibert, and W. Unger. Towards the notion of stability of approximation for hard optimization tasks and the traveling salesman problem. Theoret. Comput. Sci., 285(1):3-24, 2002.

[9] L. Sunil Chandran and L. Shankar Ram. Approximations for ATSP with parametrized triangle inequality. In Proc. 19th Int. Symp. on Theoret. Aspects of Comput. Sci. (STACS), volume 2285 of Lecture Notes in Comput. Sci., pages 227-237, 2002.

[10] Nicos Christofides. Worst-case analysis of a new heuristic for the travelling salesman problem. In J. F. Traub, editor, Algorithms and Complexity: New Directions and Recent Results, page 441. Academic Press, 1976.

[11] A. M. Frieze, G. Galbiati, and F. Maffioli. On the worst-case performance of some algorithms for the asymmetric traveling salesman problem. Networks, 12(1):23-39, 1982.

[12] H. Kaplan, M. Lewenstein, N. Shafrir, and M. Sviridenko. Approximation Algorithms for Asymmetric TSP by Decomposing Directed Regular Multigraphs. In Proc. 44th IEEE Symp. on Foundations of Comput. Sci. (FOCS), pages 56-65, 2003. 